\title{
Consanguineous familial study revealed biallelic FIGLA mutation associated with premature ovarian insufficiency
}

\author{
Beili Chen ${ }^{1 \dagger}$, Lin Lii ${ }^{2+}$, Jing Wang ${ }^{3}$, Tengyan Li ${ }^{4}$, Hong Pan ${ }^{4}$, Beihong Liư ${ }^{4}$, Yiran Zhou', Yunxia Cao ${ }^{1,5,6^{*}}$ \\ and Binbin Wang ${ }^{1,4,7^{*}}$
}

\begin{abstract}
Background: To dissect the genetic alteration in two sisters with premature ovarian insufficiency (POI) from a consanguineous family.

Methods: Whole-exome sequencing technology was used in the POI proband, bioinformatics analysis was carried out to identify the potential genetic cause in this pedigree. Sanger sequencing analyses were performed to validate the segregation of the variant within the pedigree. In silico analysis was also used to predict the effect and pathogenicity of the variant.

Results: Whole-exome sequencing analysis identified novel and rare homozygous mutation associated with POI, namely mutation in FIGLA (c.2 T>C, start codon shift). This homozygous mutation was also harbored by the proband's sister with $\mathrm{POI}$ and was segregated within the consanguineous pedigree. The mutation in the start codon of the FIGLA gene alters the open reading frame, leading to a FIGLA knock-out like phenotype.

Conclusions: Biallelic mutations in FIGLA may be the cause of POI. This study will aid researchers and clinicians in genetic counseling of POI and provides new insights into understanding the mode of genetic inheritance of FIGLA mutations in POl pathology.
\end{abstract}

Keywords: Premature ovarian insufficiency, FIGLA, Whole-exome sequencing

\section{Background}

Premature ovarian insufficiency (POI) is a severe disorder of ovarian dysfunction and is characterized as decreased ovarian reserve and increased folliclestimulating hormone (FSH) level. The aetiology is complex, among which the genetic alteration is one of the causes of POI and includes X chromosomal abnormalities, balanced translocations, and fragile $\mathrm{X}$ mental retardation 1 (FMR1) premutations and single gene defects [1]. Although several single gene variants have been associated with POI in recent decades [1], only a few have been proven to cause POI, e.g., FSHR, BMP15, NOBOX, MCM8, MCM9, STAG3, HFM1, MSH4, and

\footnotetext{
*Correspondence: caoyunxia_profr@126.com; wbbahu@163.com

${ }^{\dagger}$ Beili Chen and Lin Li contributed equally to this work.

'Department of Obstetrics and Gynecology, Reproductive Medicine Center,

The First Affiliated Hospital of Anhui Medical University, Meishan Road,

Shushan, Hefei 230022, China

Full list of author information is available at the end of the article
}

MSH5 [2]. Recently, we and another groups suggested that some genes and mutations cause POI in an autosomal recessive mode of inheritance rather than previous suggested heterozygous manner [3-6]. Together, these findings have expanded our knowledge on POI and helped distinguish causative mutations and risk alleles for POI.

In recent years, whole-exome sequencing (WES) technology has become a powerful tool for elucidating the genetic causes of familial POI, especially in consanguineous pedigrees [2]. In this study, we used WES to identify a novel mutation for POI in a consanguineous family. Our study is the first to report that mutations in FIGLA cause POI in a recessive manner. Our findings will aid researchers and clinicians to understand genetic causes for POI and help patients by genetic counseling.

(c) The Author(s). 2018 Open Access This article is distributed under the terms of the Creative Commons Attribution 4.0 International License (http://creativecommons.org/licenses/by/4.0/), which permits unrestricted use, distribution, and 
a

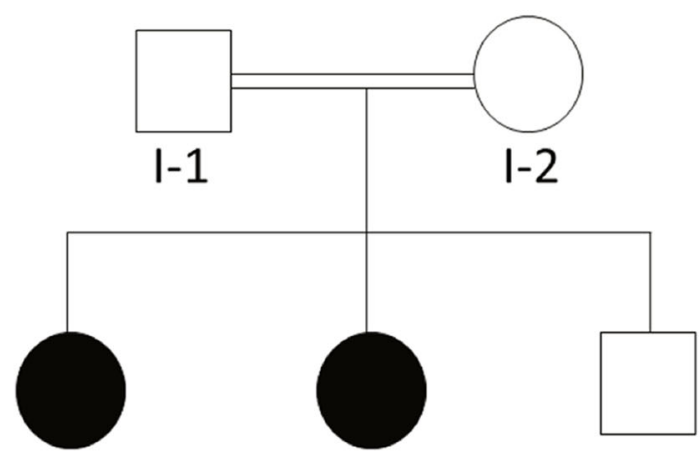

II-1 II-2 II-3

b

\section{c. $2 \mathrm{~T}>\mathrm{C}$}

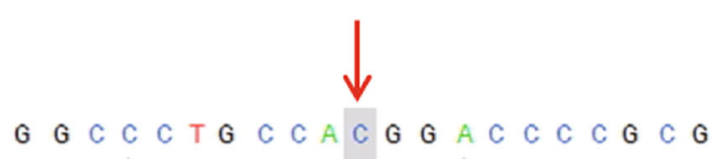

Proband II-1

Affected

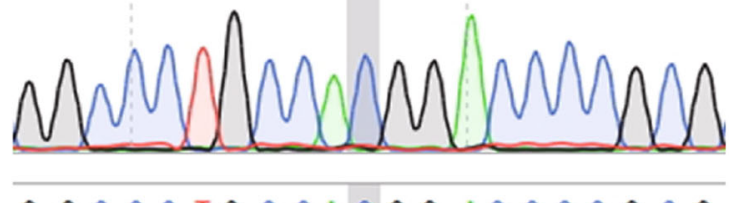

Proband's sister II-2 Affected

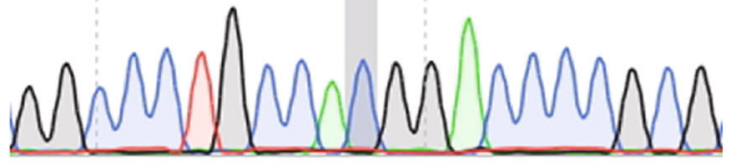

$G G C C C T G C C A T G G A C C C C G C G$

Proband's father I-1

Unaffected

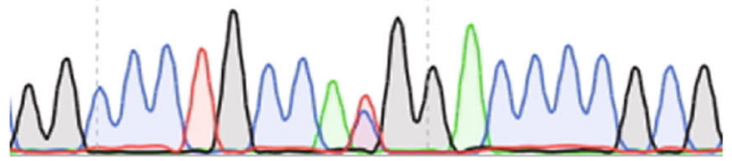

G G C C C T G C C A T G G A C C C C G C G

Proband's mother I-2

Unaffected

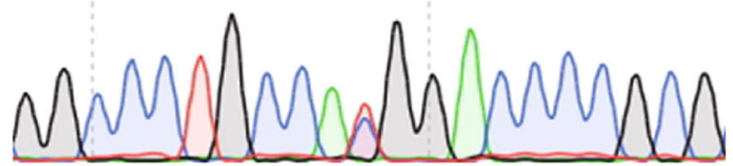

Fig. 1 Pedigree and genetic analysis of the patients in family 1. (a) Two POI patients in a Chinese consanguineous pedigree. The black circle indicates the affected family members. (b) Sanger sequencing validation of the mutation in family members. Red arrow indicates the mutation site 
Table 1 In silico analysis of the FIGLA mutation

\begin{tabular}{|c|c|c|c|c|c|c|c|c|c|c|}
\hline Gene & Mutation & $\begin{array}{l}\text { Amino acid } \\
\text { change }\end{array}$ & Consanguinity & Zygosity & $\begin{array}{l}\text { Mutation } \\
\text { Taster }^{a}\end{array}$ & SNPS\&GO & FATHMM-MKL ${ }^{c}$ & $\begin{array}{l}\text { ExAC } \\
(\text { total) }\end{array}$ & $\begin{array}{l}\text { ExAC } \\
\text { (East Asian) }^{\mathrm{e}}\end{array}$ & $\begin{array}{l}\text { gnomAD } \\
\text { (total) }^{f}\end{array}$ \\
\hline$F I G L A$ & c. $2 \mathrm{~T}>\mathrm{C}$ & Start ATG shift & Yes & Homozygous & $\begin{array}{l}\text { Disease causing } \\
\text { (1) }\end{array}$ & $\begin{array}{l}\text { Neutral } \\
(0.157)\end{array}$ & $\begin{array}{l}\text { Damaging } \\
(0.8119)\end{array}$ & 0 & 0 & 0 \\
\hline
\end{tabular}

${ }^{a}$ Mutation Taster (http://www.mutationtaster.org/). The probability value is the probability of the prediction, i.e., a value close to 1 indicates a high 'security' of the prediction

${ }^{b}$ SNPs\&GO (http://snps.biofold.org/snps-and-go/). Disease probability (if $>0.5$ mutation is predicted Disease)

${ }^{\mathrm{C}} \mathrm{FATHMM-MKL}$ (http://fathmm.biocompute.org.uk/fathmmMKL.htm). Values above 0.5 are predicted to be deleterious, while those below 0.5 are predicted to be neutral or benign

${ }^{d}$ Frequency of variations in total of ExAC database.

${ }^{\mathrm{e}}$ Frequency of variations in East Asian population of ExAC database

${ }^{f}$ Frequency of variation in total of gnomAD (genome Aggregation Database, a big database containing 123,136 exome sequences and 15,496 whole-genome sequences)

\section{Methods}

\section{Patients}

Two sisters with POI were recruited from The First Affiliated Hospital of Anhui Medical University. Their parents were consanguineous marriage. POI was diagnosed if the patients had amenorrhea for at least 4 months under the age of 40 and two consecutive follicle stimulating hormone (FSH) measurements $>25 \mathrm{IU} / \mathrm{L}$ taken 4 weeks apart [7]. None of the patients showed any of the following: karyotypic abnormality, autoimmune disorder, history of radiotherapy and chemotherapy, or pelvic surgery. Before the patients came to our hospital, they had been on hormone replacement therapy. This study was approved by the Ethics Committee of Anhui Medical University. Written informed consent was obtained from all participants, and peripheral blood $(5 \mathrm{~mL})$ was subsequently collected from each participant.

\section{WES and sanger sequencing validation}

WES was performed as previously described [8]. WES was carried out on the proband. The sequencing raw reads were 32,530,181, and the raw depth $(x)$ was 161 . 44. Sanger sequencing was performed using genespecific primers. Firstly, the PCR product containing the FIGLA's mutation site was amplified using the specific primers as follow, 5'-GCGAGAAAGAGGGCAACG-3' for forward primer, and 5'-TGGTGGTAGAGCAGG GAAGG-3' for reverse primer.

\section{Bioinformatic analysis of the sequence variant}

Three online prediction tools were used in this study to predict the pathogenicity of the variant. For Mutation Taster (http://www.mutationtaster.org/), the probability value is the probability of the prediction, i.e., a value close to 1 indicates a high 'security' of the prediction. For SNPs\&GO (http://snps.biofold.org/snps-and-go/), if the value was more than 0.5 , the mutation is predicted as Disease. For FATHMM-MKL (http://fathmm.biocompute.org. uk/fathmmMKL.htm), values above 0.5 are predicted to be damaging, while those below 0.5 are predicted to be neutral or benign. The allele frequency was evaluated by searching the human exome-seq or whole genome-seq databases such as Exome Aggregation Consortium (ExAC, http:// exac.broadinstitute.org/) and genome Aggregation Database (gnomAD, http://gnomad.broadinstitute.org/). ExAC contained 60,706 unrelated individuals' exome sequencing data, and gnomAD is a big database containing 123,136 exome sequences and 15,496 whole-genome sequences.

\section{Results \\ Clinical features of the family}

Both the proband (II-1, Fig. 1a) and her younger (II-2) were diagnosed as POI with primary amenorrhea. The proband's father and mother were in a consanguineous marriage. The proband's paternal grandfather and maternal grandmother were siblings. The proband was 31 years old when diagnosed with POI in 2011. Her height was $172 \mathrm{~cm}$ and weight was $75 \mathrm{~kg}$. Hormone levels of the proband were as follows: FSH, $36.81 \mathrm{IU} / \mathrm{L}$; luteinizing hormone (LH), $10.2 \mathrm{IU} / \mathrm{L}$; testosterone (T), $1.47 \mathrm{nmol} / \mathrm{L}$; estradiol (E2), $122 \mathrm{pmol} / \mathrm{L}$; prolactin (PRL), $8.8 \mathrm{ng} / \mathrm{mL}$; and anti-Müllerian hormone (AMH), $0.05 \mathrm{ng} / \mathrm{mL}$. The proband's ovaries were not detected by transvaginal color Doppler ultrasound examination. The hormone levels of the proband's sister were as follows: FSH, 54. $49 \mathrm{IU} / \mathrm{L} ; \mathrm{LH}, 16.29 \mathrm{IU} / \mathrm{L} ; \mathrm{T}, 0.43 \mathrm{nmol} / \mathrm{L} ; \mathrm{E} 2,14 \mathrm{pmol} / \mathrm{L}$; and PRL, $10.46 \mathrm{ng} / \mathrm{mL}$. The proband's mother (I-2) underwent menopause at the age of 53. The proband's brother (II-3) was fertile and had twin daughters.

\section{Whole-exome sequencing analysis}

To identify the genetic cause for POI in these two sisters, we first set an analysis pipeline. Each patient underwent WES, with sequence reads aligned and variants called, as previously described [8]. Pedigree analysis suggested a recessive mode of inheritance. Likely causative mutations were identified after filtering polymorphisms with allele frequencies $>1 \%$ in Exome Aggregation Consortium (ExAC), Genome Aggregation Database (gnomAD), 1000 Genomes and dbSNP databases. A list of genes harboring homozygous variants was further filtered by the functional impact of the mutation, e.g., conservation and functional prediction. Gene relevance for 
a

original gDNA CTGCCATGGACCCCGCGCCCGGCGTCC

sequence snippet

altered gDNA CTGCCACGGACCCCGCGCCCGGCGTCC

sequence snippet

original cDNA CTGCCATGGACCCCGCGCCCGGCGTCC

sequence snippet

altered CDNA CTGCCACGGACCCCGCGCCCGGCGTCC

sequence snippet

wildtype AA MDPAPGVLDP RAAPPALLGT PQAEVLEDVL REQFGPLPQL ABVCRLKRLP SGGYSSTENL

sequence QLVLERRVA NAKERERIKN LMRGFARLKA LVPFLPQSRK PSKVDILKGA TEYIQVLSDL

LEGAKDSKKQ DPDEOSYSMN SSESHTSSAR OLSRNITOHI SCAFGLKNEE EGPUADGGSG

EPAHACRHSW MSTTEIISPT RSLDRFPEVE LLSHRLPQV*

mutated AA MSRAIVTTVL NHIHPRQDSC QETSPNISAV LSA*

sequence

b

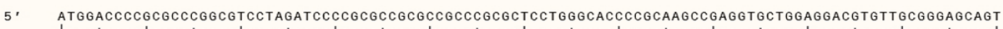

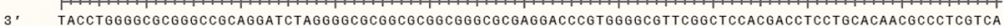
Met Asp Pro Ala Pro Gly Val Leu Asp Pro Arg Ala Ala Pro Pro Ala Leu Leu Gly Thr Pro Gln Ala Glu Val Leu Glu Asp Val Leu Arg Glu Gln

TCGOGCCGCTOCCCCAGCTGOCCOCTOTCTOCCGGCTCAAGCGOCTOCCCTCGOGCOGCTACTCGTCCACTGAAAACCTCCAGTTOGTOCTOGAGCGGCO

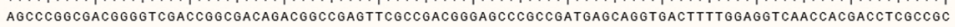
phe Gly pro Leu pro Gin Leu Ala Ala Val Cys Arg Leu Lys Arg Leu pro Ser Gly Gly Tyr Ser Ser Thr Glu Asn Leu Gin Leu Val Leu Glu Arg Arg -

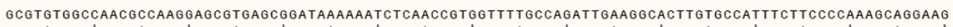

CGCACACCGGTTGCGGTTCCTCOCACTGOCCTATTTITTAGAGTTGGACCAAAACGGTCTAACTTCGGTGAACACGGTAAGGAGGGGTTTCGTCCTTC Arg Val Ala Asn Ala Lys Glu Arg Glu Aro Ile Lys Asn Leu Asn Arg Gly Phe Ala Arg Leu Lys Ala Leu Val Pro Phe Leu Pro Gln Ser Arg Lys

CCCAGCAAAGTTGATATCCTTAAAGGTOCGACTGAATATATACAGGTTCTCAGTGATCTTTIGGAAGGAGCCAAAGACTCAAAGAAACAAGACCCAGATG GgGTCGTTCAACTATAGgaATITCCACGCTGACTTATATATGTCCAAGAGTCACTAGAAAACCTTCCTCGGTTTCTGAGTTTCTITGTTCTGGGTCTAC Pro Ser Lys Val Asp tle Leu Lys Gly Ala Thr Glu Tyr te Gln Val Leu Ser Asp Leu Leu Glu Gly Ala Lys Asp Ser Lys Lys Gln Asp Pro Asp

AgCagagCTATAGTAACAACAGTTCTGAATCACATACATCCTCGOCAAGACAGCTGTCAAGAAACATCACCCAACATATCAGCTOTGCTTTCGGCTTGAA TCGTCTCGATATCATTGTTGTCAAGACTTAGTGTATGTAGGaGCCGTCTGTCGACAGTTCTTTGTAGTGGGTTGTATAGTGGACACGAAAGCGGAACTT Glu Gin Ser Tyr Ser Asn Asn Ser Ser glu Ser His Thr Ser Ser Ala Arg Gin Leu Ser Arg Asn tle Thr Gln His tle Ser Cys Ala phe gly leu lys GAATGAAGAGGAAGGGCCTTGGGCAGATGGTGGCAGTGGTGAGCCAGCACACGCTTGTCGCCACAGTGTGATGTCTACGACTGAAATTATCTCCCCAACC CTTACTICTCCTTCCCGGAACCCGTCTACCACCGTCACCACTCGGTCGTGTGCGACAGCGGTGTCACACTACAGATGCTGACTITAATAGAGgGGTTGG Asn Glu Glu Giu Gly Pro Trp Ala Asp Gly Gly Ser Gly Glu Pro Ala His Ala Cys Arg His Ser Val Met Ser Thr Thr Glu lle lle Ser Pro Thr

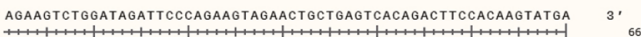

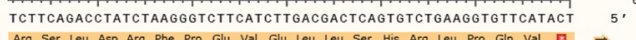
Arg Ser lou Asp Arg Phe Pro Glu val Glu lou lou Ser His Arg leu Pro Gin Val $\square \rightarrow$

C

5. ACGGACCCCGCGCCCGGCGTCCTAGATCCCCGCGCCGCGCCGCCCGCGCTCCTGGGCACCCCGCAAGCCGAGGTGCTGGAGGACGTGTTGCGGGAGCAGTTCGGGCCGC

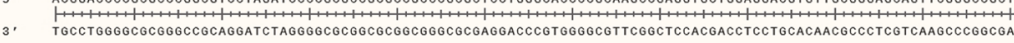

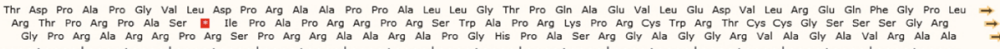

GCCCCAGCTGGCCGCTGTCTGCCGGCTCAAGCGGCTGCCCTCGGGCGGCTACTCGTCCACT GAAAACCTCCAGTTGGTGCTGGAGCGGCGGCGTGT GGCCAACGCCAAGO

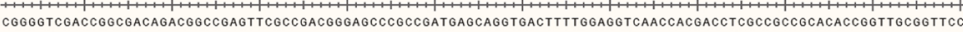

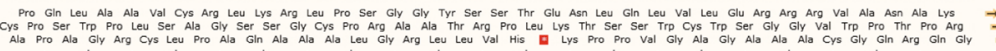

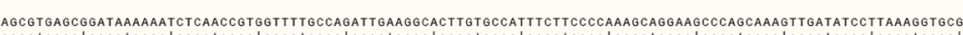
TCGCACTCGCCTATTITTTAGAGT TGGCACCAAAACGGTCTAACTTCCGTGAACACGGTAAAGAAGGGGTTTCGTCCTTCGGGTCGTTTCAACTATAGGAATITCCACGC

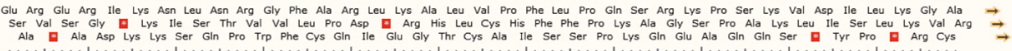

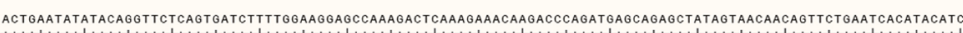
TGACTTATATATGTCCAAGAGTCACTAGAAAACCTTCCTCGGTTTCTGAGTTTCTTTGTTCTGGGTCTACTCGTCTCGATATCATTGTTGTCAAGACTTAGTGTATGTAC

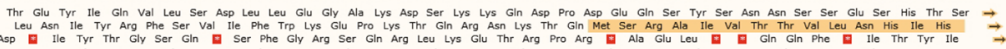

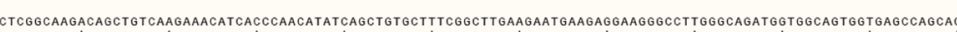
GAGCCGTTCTGTCGACAGTTCTTIGTAGTGGGTTGTATAGTCGACACGAAAGCCGAACTTCTTACTTCTCCTTCCCGGAACCCGTCTACCACCGTCACCACTCGGTCGTG

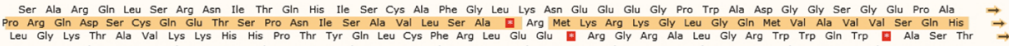

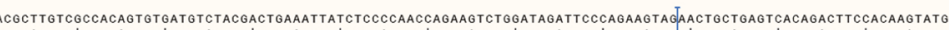

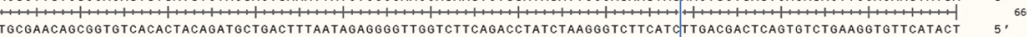

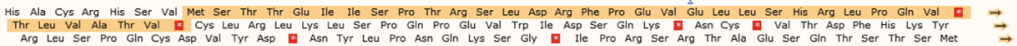

Fig. 2 (See legend on next page.) 
(See figure on previous page.)

Fig. 2 (a) In silico analysis of the alternative start site and potential resulting protein. The MutationTaster online prediction tool (http:// www.mutationtaster.org/) was used in the current analysis. The predicted results for c.2 T > C showed that the start ATG shifted, the Kozak consensus sequence changed, and the entire protein amino acid sequence frameshifted and was changed. The green box indicates the wild-type amino acid sequence, while the red box indicates the mutated amino acid sequence. (b) and (c) The wild-type (b) and mutated (c) sequences of FIGLA. The light grown indicate the putative translated protein sequences

ovarian function was also assessed using gene expression data (Human Protein Atlas, http://www.proteinatlas. org/) and mouse model phenotype data (Mouse Genome Informatics, http://www.informatics.jax.org/).

According to pipeline and several filtering steps after WES, only the FIGLA (folliculogenesis specific bHLH transcription factor) gene and its homozygous mutation, NM_001004311:exon1:c.2 T > C, was identified in the proband. This homozygous mutation was also harbored by the proband's sister and was segregated within the pedigree (Fig. 1b). FIGLA has been shown to be specifically expressed in human ovary $[9,10]$ and was reported to be associated with POI by haploinsufficiency effect [11]. However, our finding did not support the haploinsufficiency effect of FIGLA causing POI.

\section{Bioinformatics analysis of the mutation}

In silico analysis by four online prediction tools suggested that the c.2 $\mathrm{T}>\mathrm{C}$ mutation was a pathogenic mutation (Table 1). The c. $2 \mathrm{~T}>\mathrm{C}$ mutation lost the start codon and therefore the open reading frame of FIGLA was altered (Fig. $2 \mathrm{a}, \mathrm{b}$ and $\mathrm{c}$ ). The c. $2 \mathrm{~T}>\mathrm{C}$ mutation was absent in the ExAC, gnomAD, 1000G and dbSNP databases, indicating the extremely rarity of this mutation.

\section{Discussion}

Here we report that a novel homozygous mutation in FIGLA is associated with POI in one consanguineous pedigree.

FIGLA encodes a basic helix-loop-helix transcription factor that regulates primordial follicle formation [12, 13]. FIGLA is specifically expressed in human and mouse ovaries [9, 10, 14]. Figla knockout mice cannot form primordial follicles, leading to oocyte loss and female sterility [13]. FIGLA transcriptionally activates oocyte-related genes, such as $Z P 1, Z P 2$ and $Z P 3[10,13]$, and represses sperm-associated genes during postnatal oogenesis [15].

A previous study found that FIGLA haploinsufficiency may be associated with premature ovarian failure [11]. The study found a heterozygous p.G6 fs*66 mutation in a patient with secondary amenorrhea at 36 years of age, and a heterozygous p.140delN mutation in a patient with secondary amenorrhea at 27 years of age [11]. The p. G6 fs*66 mutation creates FIGLA haploinsufficiency, because the truncated protein only shares five amino acids with the wild-type protein [11]. The p.140delN mutation disrupts FIGLA binding to TCF3 [11], however no dominant negative effect of p.140delN was observed in that study. So that study did not prove the cause and effect of the variants. While our findings indicate a novel underlying mechanism based on two facts: one is that, the patient's mother, who is heterozygous for the mutation, was neither a POI patient nor had the phenotype of premature menopause; another is that, the c. $2 \mathrm{~T}>\mathrm{C} \mathrm{mu}$ tation abolishes the start codon, leading to an alternative open reading frame. The two facts above indicated that loss of function in one FIGLA allele can not cause POI. Therefore, our consanguineous pedigree analysis suggests that recessive inheritance of the FIGLA mutation is implicated in POI pathogenesis. However, our study did not oppose the previous finding, as the patient with homozygous mutation in our study was primary amenorrhea while the patient with heterozygous mutation in the previous study was secondary amenorrhea. Based on the current results and those of previous studies, we hypothesized that FIGLA haploinsufficiency may cause a milder POI than the FIGLA homozygous allele mutations.

\section{Conclusions}

Taken together, our study demonstrates biallelic FIGLA mutation causing POI with primary amenorrhea. This study will aid researchers and clinicians in genetic counseling of POI and provides new insight into understanding the mode of genetic inheritance of certain genes and POI.

\section{Acknowledgments}

We thank Rachel James, Ph.D., from Edanz Group (www.edanzediting.com/ ac) for editing a draft of this manuscript.

Conflict of interest

All authors declare no conflict of interest.

\section{Funding}

Research funding was provided by the National Natural Science Foundation of China (81701405) and the National Research Institute for Family Planning (2017GJZ05).

\section{Authors' contributions}

Beili Chen carried out the clinical sample collection and clinical diagnosis. Lin Li provided the manuscript preforming and carried out experiments preforming. Jing Wang and Hong Pan carried out the manuscript revision and the data analysis. Tengyan Li, Beihong Liu and Yiran Zhou participated in the experiments preforming. Yunxia Cao and Binbin Wang conceived the study. All authors read and approved the final manuscript.

Ethics approval and consent to participate

The study protocol was in accordance with the tenets of the Declaration of Helsinki and was approved by Anhui Medical University's ethics committee. 


\title{
Publisher's Note
}

Springer Nature remains neutral with regard to jurisdictional claims in published maps and institutional affiliations.

\begin{abstract}
Author details
'Department of Obstetrics and Gynecology, Reproductive Medicine Center, The First Affiliated Hospital of Anhui Medical University, Meishan Road, Shushan, Hefei 230022, China. ${ }^{2}$ Central Laboratory, Beijing Obstetrics and Gynecology Hospital, Capital Medical University, Chaoyang, Beijing 100026, China. ${ }^{3}$ Department of Medical Genetics and Developmental Biology, School of Basic Medical Sciences, Capital Medical University, No. 10 Xitoutiao, Youanmenwai, Fengtai, Beijing 100069, China. ${ }^{4}$ Center for Genetics, National Research Institute for Family Planning, 12 Dahuisi Road, Haidian, Beijing 100081, China. ${ }^{5}$ Institute of Reproductive Genetics, Anhui Medical University, Meishan Road, Shushan, Hefei 230032, China. ${ }^{6}$ Anhui Provincial Engineering Technology Research Center for Biopreservation and Artificial Organs, Meishan Road, Shushan, Hefei 230027, China. ${ }^{7}$ Key Laboratory of Family planning and Reproductive Genetics, National Health and Family Planning Commission, Heb Research institute For Family Planning, Beijing 050071, People's Republic of China.
\end{abstract}

Received: 15 January 2018 Accepted: 8 May 2018

Published online: 18 June 2018

\section{References}

1. Qin Y, Jiao X, Simpson JL, Chen ZJ. Genetics of primary ovarian insufficiency: new developments and opportunities. Hum Reprod Update. 2015, 21:787-808.

2. Laissue $P$. The molecular complexity of primary ovarian insufficiency aetiology and the use of massively parallel sequencing. Mol Cell Endocrinol. 2017;

3. Li L, Wang BB, Zhang W, Chen BL, Luo MN, Wang J, Wang X, Cao YX, Kee K. A homozygous NOBOX truncating variant causes defective transcriptional activation and leads to primary ovarian insufficiency. Hum Reprod. 2017;32:248-55.

4. Mayer A, Fouquet B, Pugeat M, Misrahi M. BMP15 "knockout-like" effect in familial premature ovarian insufficiency with persistent ovarian reserve. Clin Genet. 2017;92:208-12.

5. Franca MM, Funari MFA, Nishi MY, Narcizo AM, Domenice S, Costa EMF, Lerario AM, Mendonca BB. Identification of the first homozygous 1-bp deletion in GDF9 gene leading to primary ovarian insufficiency by using targeted massively parallel sequencing. Clin Genet. 2017;

6. Franca MM, Funari MFA, Lerario AM, Nishi MY, Pita CC, Fontenele EGP, Mendonca BB. A novel homozygous 1-bp deletion in the NOBOX gene in two Brazilian sisters with primary ovarian failure. Endocrine. 2017;58:442-7.

7. Webber L, Davies M, Anderson R, Bartlett J, Braat D, Cartwright B, Cifkova R, de Muinck K-SS, Hogervorst E, Janse F, et al. ESHRE guideline: management of women with premature ovarian insufficiency. Hum Reprod. 2016;31:926-37.

8. Wang B, Li L, Zhu Y, Zhang W, Wang X, Chen B, Li T, Pan H, Wang J, Kee K, Cao Y. Sequence variants of KHDRBS1 as high penetrance susceptibility risks for primary ovarian insufficiency by mis-regulating mRNA alternative splicing. Hum Reprod. 2017;32:2138-46.

9. Huntriss J, Gosden R, Hinkins M, Oliver B, Miller D, Rutherford AJ, Picton HM. Isolation, characterization and expression of the human factor in the germline alpha (FIGLA) gene in ovarian follicles and oocytes. Mol Hum Reprod. 2002;8:1087-95.

10. Bayne RA, Martins Da Silva SJ, Anderson RA. Increased expression of the FIGLA transcription factor is associated with primordial follicle formation in the human fetal ovary. Mol Hum Reprod. 2004;10:373-81.

11. Zhao H, Chen ZJ, Qin YY, Shi YH, Wang S, Choi Y, Simpson JL, Rajkovic A. Transcription factor FIGLA is mutated in patients with premature ovarian failure. Am J Hum Genet. 2008:82:1342-8.

12. Pangas SA, Rajkovic A. Transcriptional regulation of early oogenesis: in search of masters. Hum Reprod Update. 2006:12:65-76.

13. Soyal SM, Amleh A, Dean J. FIGalpha, a germ cell-specific transcription factor required for ovarian follicle formation. Development. 2000;127:4645-54.

14. Liang L, Soyal SM, Dean J. FIGalpha, a germ cell specific transcription factor involved in the coordinate expression of the zona pellucida genes. Development. 1997;124:4939-47.

15. Hu W, Gauthier L, Baibakov B, Jimenez-Movilla M, Dean J. FIGLA, a basic helix-loop-helix transcription factor, balances sexually dimorphic gene expression in postnatal oocytes. Mol Cell Biol. 2010;30:3661-71.

\section{Ready to submit your research? Choose BMC and benefit from:}

- fast, convenient online submission

- thorough peer review by experienced researchers in your field

- rapid publication on acceptance

- support for research data, including large and complex data types

- gold Open Access which fosters wider collaboration and increased citations

- maximum visibility for your research: over $100 \mathrm{M}$ website views per year

At BMC, research is always in progress.

Learn more biomedcentral.com/submissions 